\title{
Digital Communication Strategies in Visceral Medicine
}

\author{
Jürgen Ferdinand Riemann ${ }^{a, b}$ Oliver G. Opitz ${ }^{c, d, e} \quad$ Axel Naumann ${ }^{f}$ \\ Ulrich Tappe $^{g}$ Andreas Teufel $^{\text {h, }}$ i Alexander Meining ${ }^{j}$
}

${ }^{a}$ Director emeritus, Department of Medicine C, Klinikum Ludwigshafen, Ludwigshafen, Germany; ${ }^{\text {bStiftung }}$ LebensBlicke, Ludwigshafen, Germany; ${ }^{c}$ Coordinating Unit for Digital Medicine Baden-Württemberg (KTBW), Heidelberg University, Mannheim, Germany; ${ }^{\mathrm{d} M a n n h e i m}$ Institute of Public Health, Social and Preventive Medicine (MIPH), Heidelberg University, Mannheim, Germany; ${ }^{\text {H} H e a l t h ~ C a r e ~ I n n o v a t i o n ~ I n s t i t u t e ~} \mathrm{GmbH}(\mathrm{HCl} 2)$, Mannheim,

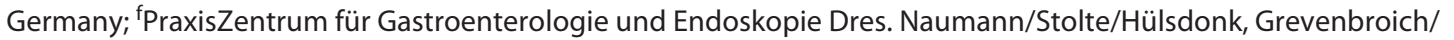
Dormagen, Germany; ${ }^{9}$ Gastro-Praxis an der St. Barbaraklinik, Hamm, Germany; ${ }^{\mathrm{h}}$ Department of Medicine II, Section of Hepatology, University Medical Center Mannheim, Medical Faculty Mannheim, Heidelberg University, Mannheim, Germany; 'Clinical Cooperation Unit Healthy Metabolism, Center for Preventive Medicine and Digital Health BadenWürttemberg (CPDBW), Medical Faculty Mannheim, Heidelberg University, Mannheim, Germany; ${ }^{\text {DDepartment of }}$ Internal Medicine II, Interventional and Experimental Endoscopy (InExEn), Gastroenterology, University Hospital Würzburg, Würzburg, Germany

\section{Riemann: How Do You See the Acceptance of Digital Communication among Health-Care Providers and Patients in Medicine?}

\section{Opitz}

Digital readiness seems very different in between the group of health-care providers and between health-care providers and patients. Digital tools, especially telemedicine, have experienced their breakthrough with the COVID-19 pandemic, as more and more physicians in practice and more and more patients preferred this line of communication. A 2020 survey among physicians in Germany, France, Italy, Spain, and Great Britain suggests that the proportion of telemedical advice and treatment in these countries will increase permanently overall - from $15 \%$ before the crisis to $34 \%$ in the future. In Germany, in a recent poll almost three-quarters (74\%) of GPs assume that in future, every fifth consultation will take place via video consultation. There are some indications that patients are even more open than healthcare providers, with respect to digital innovation. But it is still obvious: there is still a lot to do for everyone involved to help digital health achieve a breakthrough in Germany.

\section{Naumann}

Digital communication among health-care providers - be it P2D, D2D, or others - is one of the key elements of modern communication with the advantages of fast data transmission, structured data archive, avoidance of duplicate examinations, and minimization of errors. Access to digital communication tools must be simple and easily accessible so that everyone involved has access to those digital communication channels. Acceptance can only grow through education and conviction. The benefits must be visible and the bureaucratic and financial effort manageable, especially for health-care providers. In principle, the acceptance of digital communication in the German health-care system is high - rather, a throughout implementation is currently still missing due to a lack of convenient solutions, usability, an extensive heterogeneity of the available systems, and the lack of digital literacy of the stakeholders.

In order to increase acceptance of digital solutions, the following implementations are essential:

1. Extensive information (campaigns) about the advantages of digital communication

2. Easy access to the digital communication system

3. No legal obligation to participate

4. No additional costs. 


\section{Tappe}

Smartphones and tablets are now common technological platforms for communication. Video calls are increasingly used for physician-patient communication. Acceptance would certainly advance even faster if there were no bureaucratic hurdles and complicated data protection requirements that do not apply in normal everyday life. Particularly, health-care providers have difficulties in observing and complying with the abundance of regulations.

\section{Teufel}

The acceptance of digital technologies can be assumed for most patients with chronic diseases. In a US survey on 40 patients with liver cirrhosis, $60 \%$ of those surveyed said that they would use a smartphone application (app) for daily monitoring of their cirrhosis. However, large and controlled surveys are lacking. Also, as smartphone games were introduced to increase the adherence for diverse indications, $84 \%$ of patients with liver cirrhosis stated that they would also use those games if they could clearly identify cognitive deterioration. Thus, the overall acceptance can be assumed to be high if patients suffer from chronic conditions, and apps promise to prevent major complications. If however, those digital tools will find broad acceptance within the general population suffering from only minor conditions or having no subjective symptoms (e.g., patients with fatty liver disease or controlled cholestatic liver disease) remains to be seen. Key to broad acceptance will certainly be an easy usability, clear data protection concept, and universal availability independent of (potentially diverse) commercial platforms. The latter is particularly dependent on well-defined data structures and interfaces. As a side note, support for the elderly and/or disabled needs to be considered in order to extend the acceptance of these tools to those populations with the highest need.

\section{Meining}

The acceptance is related to the measures performed to enable safe data transfer. In addition, digital communication may not be too complex and time-consuming. Hence, we need safe but easy and intuitive security measurements. If these are enabled, I would expect a high acceptance from both sides, the patients and health-care providers.

\section{Riemann: Are Apps on Prescription a Viable Option in Doctor-Patient Communication?}

\section{Opitz}

Through the "Digital Health Care Act" (DVG), the socalled "DiGAs," digital health applications can now be viewed as standard care for those with statutory health insurance in Germany. These applications - apps or browser-based - upon application by the DiGA provider are evaluated by the Federal Institute for Drugs and Medical Devices (BfArM) on the basis of a publicly available catalog of criteria and, if the evaluation is positive, are temporarily or permanently listed as reimbursable DiGAs. It remains to be seen how these DiGAs (15 as of June 1st) are now accepted and prescribed by treating physicians. As with any new therapeutic option, the DiGAs are one tool in the arsenal of options a treating physician has in supporting "his" patients. As an option, it will not replace other options nor will it replace the face-to-face communication between the patient and "his" physician. But it certainly can be a complimentary tool, positively influencing outcomes in terms of medical benefits or in positive health-care effects.

\section{Naumann}

Digital applications will certainly make their way into various health-care areas in the coming years. To what extent the current first generation of digital health applications (DiGAs) in Germany already exhibits the full technological and medical potential remains to be seen over the coming years and will be measured by means of acceptance by patients and medical providers. It will certainly be crucial that the additional benefit for the patient is scientifically proven in order to distinguish valuable therapeutic, therapy-supporting, or diagnostic applications from "lifestyle" products. The potential is huge, and some of the possibilities that will arise can only be guessed at. However, again, it will be crucial to convince users of the application and to generate added value for all participants.

\section{Tappe}

Prescription apps may be a reasonable option. Many providers are currently entering the market. It will be the crucial to separate the valuable from nonuseful tools. Good applications may then offer helpful support and, if necessary, support the patient's independence or make data for treatment available to the practitioner.

\section{Teufel}

Health apps may now be prescribed by physicians in Germany under the Digital Health Care Act. As the field is still beginning to emerge, the full range of support for a viable doctor-patient communication remains to be seen. However, among wishes and expectations of patients regarding app development, communication with their physician was reported to be of highest importance to patients. However, the development of this field is generally not only dependent on the medical need but also - since currently the primary used platform is smartphones - on the technological capabilities of smart- 
phones. However, with new functions such as the recording of pulse and ECG, steps, sleep behavior, and other options, smartphones provide increasingly useful features for patient monitoring and also doctor-patient communication.

With respect to doctor-patient communication, currently developed and available apps may offer support, especially for taking medical history, for example, by systematically collecting information on gastrointestinal symptoms and summarizing them in a report to the treating physician. As a result, the physician has a comprehensive overview prior to the first patient contact. A field of continuous monitoring of essential physiological parameters, which is also interesting for the doctor-patient relationship, depends largely on the technical development of compatible biosensors and their connectivity with health-care providers. With the Apple HealthKit and the Samsung Health app, considerable progress is currently being made and can be expected to contribute to routine care in the next few years. Other wishes by patients such as receiving notifications concerning their medication, transmission of diagnostic results, and appointment reminders, and information regarding their clinical status, may also well be addressed in the near future. However, for these developments as well, a standardization of data structure, a well-defined data protection concept, and universal compatibility to the diverse practice software systems are essential.

\section{Meining}

An app can be a diagnostic tool similar to any medical device. Hence, there is no reason why apps on prescription are not viable. However, it should be considered that a drug, for example, undergoes several steps before it can be approved (from phase I to III). Hence, the question is how and by whom apps will be tested before they may be prescribed. The Medical Device Regulation plays certainly a role with respect to these aspects. However, the evaluation of disease-specific apps should also be formally tested and approved by our professional societies. These societies know the clinical demand and have experts that may judge an such devices.

\section{Riemann: What Are Key Issues in Implementing Digital Communication in Medicine?}

\section{Opitz}

Digitization has been given a tremendous boost by the development of the legal, technical, and social framework conditions in the recent past but most dramatically by an unexpected pandemic. In the future, evidence for positive health-care effects or positive medical benefits is equally important. Not to forget the gatekeepers or en- ablers of such new concepts of reimbursed health apps: without a prescription by physicians (general practitioner or specialist) or by psychotherapists, the new DiGA concept, for example, will not be crowned with success. Therefore, knowledge about and acceptance of DiGAs and their positive effects on the side of physicians and psychotherapists are of critical importance for the implementation of reimbursed DiGAs. Overall, to continuously implement digital innovations into the health-care system, the translation process has to focus on patient's needs and ensure early integration of all relevant stakeholder in the process.

\section{Naumann}

The key to success in the implementation of digital communication is very basic: simple and low-threshold access to the means and channels of communication, stable network infrastructure, high usability of the communication systems, and, of course, the high demands on data protection, which must be clearly defined by the legislator. Digital communication will only be used extensively if a legal framework provides legal security on all communicating levels.

\section{Tappe}

Everything new does not necessarily have to offer an improvement at initiation. The key issues are the ease of use and a clear definition of benefit for the patient. Furthermore, the technological basis is also important, as a cell phone with no reception is of little use. The expansion of network structures is therefore a basic requirement for digital communication.

\section{Teufel}

Key issues in implementing digital communication are of technological legislature but also financial nature. Generally, a major investment on wireless technology will be necessary. Particularly, real-time big data streaming for continuous data transmission and surveillance will require stable, fast, and ubiquitously available wireless connections. As stated before, the wearable and tracking technology is making progress, and the use of these tools now needs to be evaluated and validated in personalized care and patient self-management. Again, in order to be successful in implementing these technological advances, they need to be flanked by a legal framework securing data privacy, regulation for the usage of data for reimbursement purposes through health insurance companies but also data usage for research. Finally, as this effort will require a significant financial investment for using such apps and technological developments most likely being passed over to patients and insurance companies, a clear reimbursement concept needs to be set in place in order to secure acceptance also on a financial basis. 


\section{Meining}

Digital communication has been part of our daily life since several years. The advantages are apparent for many reasons, such as better organization of examinations, control of therapy effects or side effects, quality assurance, and better access to treatment relevant data, just to name some examples. Nevertheless, health data are critical. Hence, measurements to assure safe data transfer must fulfill the highest level of security, and patients and physicians must be trained on how to handle such critical data. Data leakage and misuse of personal, medical data would be a catastrophe.

\section{Riemann: What Does It Take to Organize a Digital Network with Efficient Interfaces but Lack of Major Financial Resources?}

\section{Opitz}

In order to implement digital innovation, institutions need first and foremost to be open for digital innovations and change of their processes, need to address their respective needs, and take a long-term health economic perspective. In this sense, the health-care market is not a classical market, and innovation and reimbursement do not happen at the same time nor at the same place. Openness to connect with others and tackle challenges in networks will also help acquire financial tools for digital transformation.

\section{Naumann}

Cost-effective setup of digital networks strongly relies on consistent hardware requirements, since infrastructure changes in short-term cycles (hardware and/or software) are expensive and inefficient.

\section{Tappe}

Financial issues then play a subordinate role if added value can be created. However, money can easily be wasted due to excessive bureaucracy, and implementation requires smart solutions. The supposedly secure networks in particular keep showing gaps. Securing these with expensive hardware will not help with the short life of the devices. Protection by means of biometric data is currently already successful with most simple devices. For example, the electronic doctor's card (eHBA) that has now been introduced in Germany as proof of identification can already be classified as a questionable investment.

\section{Teufel}

Key to a successful digital network under limited financial resources certainly is a well-defined medical, technological, legal, and financial framework. The use (and electronic implementation) of practice guidelines needs to be standardized. As for technological prerequisites, well-defined standards in data structure and technological compatibility among different providers will reduce costly programming efforts to transfer data between different (commercial) platforms. Again, this issue is closely correlated with legal definitions of data structure and privacy. Even more, central repositories, for example, for drug prescriptions or medical history, may also significantly reduce work, cost, and also (medication) errors. They would furthermore prevent isolated applications of medical documentation and data repositories. If the upcoming electronic health record may cover some of these aspects remains to be seen. However, some of the technological issues have already been solved to facilitate reimbursement of physicians by the health insurance companies. Secured data connections and standards for invoice transmission may in many cases and from different providers be used and may be regarded as a first step into the era of digital communication in medicine.

\section{Meining}

It takes health-care providers and patients to be willing to accept the advantages of digitalization. At the end, it is a matter of individuals. Nevertheless, there are many, particularly young people, who are grown up in a digital world. These people will organize efficient networks by themselves simply because they want to do so. However, what they need is a safe and working platform. Here, the professional societies are requested for support. During the pandemic, we have learned very fast who important digitalization is for exchange of knowledge. Each professional society was suddenly forced to change to fully digital communication platforms. The same should now be possible for digital communication among health-care providers and patients.

\section{Riemann: Which Aspects of Digitalization in Medicine Still Have a Particular Need for Research, Informatics, Medical Standards, or Public Health?}

\section{Opitz}

To achieve an entry into the DiGA directory, DiGA providers must submit a comprehensive evaluation concept, which needs to be supplied together with the initial application. In this context, the proof of real-world evidence from the actual health-care context, in which the DiGA is implemented, will be key in the future: only through the provision of positive health-care effects or positive medical benefits, DiGAs can be permanently included in the DiGA directory and thus remain reimbursable. This evidence will be the measuring stick for digital innovations. DiGAs will require the support of academic institutions to obtain the proof of positive health-care ef- 
fects or medical benefits, that is, in areas of preparing the evaluation concept, the scientific evaluation of the study designs, conducting the respective clinical trials, and thus the generation of the evidence. Academic research needs to come up with new tools and methodologies to generate evidence of digital innovations in the real world.

\section{Naumann}

The need to implement digital research exists at all levels. Artificial intelligence and neural networks for diagnostic and therapeutic support but also in drug development should certainly be mentioned at the top. As a result, medical standards will change and must be checked and validated as soon as those novel strategies are available. Central network structures will be of significant aid in implementing international committees and research groups taking care of these issues. The analysis of big data in medicine has already led to multitude of new findings and will provide even more new insights in the future. It is important to make these usable for research, care, and prevention. This requires an ethics basis, which must also be developed.

Another central research area will be data collection using wearables. What are potential benefits of a continuous collection and evaluation of data and what possibilities do they have to change the health-care system toward more prevention and not predominantly just a reaction to existing diseases? What advantages does it bring to the individual on the one hand side and to society and the health-care system on the other hand side. To answer those questions, the respective health-care research should be based on the 4 Ps: preventive, personalized, predictive, and participatory.

\section{Tappe}

The so-called artificial intelligence will certainly have the greatest need for research and development. Through sophisticated programming, machines may be trained to define statistical differences, which then lead to new insights. This is certainly an exciting field that will still offer a lot in the future. Further findings will emerge in the area of big data, where large amounts of data can be processed quickly. Again, it is necessary that these already available large amounts of data, for example, from health insurance companies, can be made available for a scientific elaboration. It is to be demanded that health data can be processed anonymously even without a lengthy discussion about individual consent to data transfer.

\section{Teufel}

Ubiquitously available, fast wireless connections are a prerequisite for the usability of digital medicine solutions. However, these may still be lacking in some (rural) areas which are in turn the areas with a potential need for tele- medicine or digital physician-patient consultations or surveillance. The progress in wearable and tracking technology now needs to be translated into clinical routine applications and clinical practice. In order to achieve this goal, those digital apps and tools will have to undergo a rigid validation in clinical trials, which need to be performed under the same standards as common drug trials (blinded, large number of patients, clear defined primary endpoint). Common data structures are also needed in order to facilitate a universal data exchange among all health-care providers independent of commercial developments and noncompatible company-owned standards. This will certainly help (iteratively) improve the field of digital technologies but also be of importance for reimbursement of those technologies by health insurance companies. Finally, for some issues such as patient history or current medication, central databases may be of help, so that all health-care providers may have easy access upon patient's approval and do not have to search numerous local or even commercial data storage repositories.

\section{Meining}

Probably all! Digitalization in medicine is an ongoing process. We should all continuously control respective devices, ways of interactions, and data safety measures.

\section{Riemann: Where Do You See a Need for Action for the Legislature?}

\section{Opitz}

The new legislation on the use of digital solutions in the health-care system and the related ordinance as well as the associated guidelines of the BfArM represent a courageous first step by the legislature to break new ground, to enable Germany to rapidly introduce digital applications in the health-care system and still have a certain regulatory effect with maintained stringency. Self-administration has also contributed to improving the framework conditions. This can and should be a blueprint for the implementation of future digital innovations.

The COVID-19 pandemic was the biggest single accelerator in bringing digital innovations to the healthcare system, and it has also shown that the implementation of digital innovation is still too slow and lacks financing tools. The legislature needs to address the legal, technical, and financial framework to accelerate that process. Additionally, the digital competencies of everybody involved will determine the success of digital innovations in the health-care market. If those issues are addressed, digital medicine can greatly contribute to better medical care and help to make the best possible use of precious medical resources. 


\section{Naumann}

Legislative considerations are required on a national and international basis. With regard to communication, there must be a clear framework with a focus on data protection and the associated legal certainty. An international code of conduct similar to the Geneva Convention must also be created. We need digital ethics for survey results and also discursive changes in all areas of being not just in health care.

An essential requirement of the legislature however must be that access to medical knowledge must be open source. With an increasing commercialization of medical knowledge and also increasing commitment of the international tech companies into the health-care sector, the risk that medical knowledge may become only available to selected or paying parts of the population significantly increases.

\section{Tappe}

Digitization will change the way we live together and, for example, also support the climate goals. The legislature must have a sense of proportion here and accompany this digital change. However, the benefits of digitization must not be developed into bureaucracy, misunderstood data protection, or demands for investments that are quickly outdated. Digitization is fast moving; the laws should be adaptable here without restricting the basic concepts.

\section{Teufel}

Key issues for the legislature are certainly to define standards but also guarantee data security. As stated before, implementing digital medicine advances need to be flanked by a legal framework securing data privacy, regulation for the usage of data for reimbursement through health insurances, and also data usage for research. Again, for some issues such as patient history or current medication, the legislature should force central repositories with highest data protection standards and well-defined data structures to facilitate easy data access for medical purposes, independent of commercial interests.

\section{Meining}

On a European level, we have already good concepts and standards. Several European professional societies are well organized under the umbrella of the Biomed Alliance, for example, and all new digital devices are evaluated by experts. From a national aspect, I see national societies to play a greater role in educating their members. Patients are flooded with diverse, sometimes unqualified and false, information. Hence healthcare professionals need skills to judge on the true benefit of these digital devices. Societies may offer certificates on digitalization in medicine to their members or at least give them some advice on the pros and cons of new devices that have been brought on the market. Here, the legislature should support the national societies logistically but also financially.

\section{Conflict of Interest Statement}

Jürgen F. Riemann: no conflict of interest to declare. Oliver G. Opitz: founder of the Health Care Innovation Institute $\mathrm{GmbH}$. Axel Naumann: no conflict of interests to declare. Ulrich Tappe: no conflict of interest to declare. Andreas Teufel: no conflict of interest to declare. Alexander Meining: received honorarium as a consultant for Olympus and Ovesco. 